\title{
Opinion Mining in Latvian Text Using Semantic Polarity Analysis and Machine Learning Approach
}

\author{
Gatis Špats and Ilze Birzniece \\ Department on Artificial Intelligence and Systems Engineering, Institute of Applied Computer Systems, \\ Riga Technical University, 1 Kalku, Riga, LV-1658, Latvia \\ gatis.spats@gmail.com, ilze.birzniece@rtu.lv
}

\begin{abstract}
In this paper we demonstrate approaches for opinion mining in Latvian text. Authors have applied, combined and extended results of several previous studies and public resources to perform opinion mining in Latvian text using two approaches, namely, semantic polarity analysis and machine learning. One of the most significant constraints that make application of opinion mining for written content classification in Latvian text challenging is the limited publicly available text corpora for classifier training. We have joined several sources and created a publically available extended lexicon. Our results are comparable to or outperform current achievements in opinion mining in Latvian. Experiments show that lexicon-based methods provide more accurate opinion mining than the application of Naive Bayes machine learning classifier on Latvian tweets. Methods used during this study could be further extended using human annotators, unsupervised machine learning and bootstrapping to create larger corpora of classified text.
\end{abstract}

Keywords: Sentiment analysis, opinion mining, semantic polarity, automatic classification of Latvian text.

\section{Introduction}

Growth of the amount of information accessible through the web and especially through social networks on the one hand and comparatively limited capabilities of the human mind to perceive and process large amounts of information on the other hand have raised the demand for ways to interpret this information automatically. By using opinion mining it is possible to analyze and aggregate information that many people post online to improve tasks like advertising efficiency analysis, social media monitoring, targeted advertising, and content filtering. Opinion mining can be described as the computer-aided analysis of people's opinions, attitudes and emotions toward some certain entity. Terms 'opinion mining' and 'sentiment analysis' in literature are commonly used interchangeably.

The majority of work and especially tools for opinion mining are focused on widely used languages such as English. The need to build resources for languages other than English is identified by several researchers. Authors of [1] performed a comprehensive study on opinion mining algorithms and applications, reviewing 54 sources, and stated that "the interest in languages other than English in this field is growing as there is still a lack of resources and researches concerning these languages". This paper is devoted to opinion mining in Latvian texts. A typical issue for less widely spoken languages is the availability of text resources for creating automated opinion mining systems. Developing these resources and applying different method combinations for opinion mining in Latvian is the scope of our research. 


\section{Related Work}

Nowadays opinion mining and sentiment analysis is a widely studied area. Active research in opinion mining started around the year 2001 [2]. According to a widely cited study by Pang and Lee, factors behind this "land rush" include (1) the rise of machine learning methods in natural language processing, (2) the availability of datasets for machine learning algorithms to be trained on, and (3) comprehension of the fascinating intellectual challenges and commercial applications in the area of opinion mining. Since then there have been hundreds of thousands of papers published on the subject (around 1.4 million articles according to Google Scholar [3] search results).

According to the authors of [4], for the majority of languages the lexical resources that dictionary-based methods need for opinion mining content in social media are insufficient. Our observations confirm their statement regarding a mismatch between the formality of many language resources, such as WordNet, and the informal language of social media.

The authors of [5] created a large knowledge graph of 7 million vocabulary words from 136 languages with over 131 million semantic interlanguage links using their propagated lexicons. They have created a lexicon with 1938 words for the Latvian language as well. During the review of this lexicon, some positive and negative sentiment words seemed ambiguous, e.g., the negative word lexicon contains words "osta" (port), "dzelzcel̦š" (railroad), "objekts" (object). This result illustrates that, although some methods of sentiment extraction seem to be 'languageagnostic', in practice results obtained still need to be carefully vetted by language speakers as the results may contain ambiguities and errors.

Based on a hypothesis that methods, problem areas and results obtained regarding opinion mining in languages other than English could be relevant to opinion mining in Latvian, we reviewed multiple non-English opinion mining studies that have been performed in recent years, particularly focusing on opinion mining of texts in Twitter social network. Accuracy of sentiment detection obtained by researchers varies: 64.9\% (Portuguese) / 79.8\% (German) / 74.9\% (French) [6], 81\% (Spanish) [7], 85.2\% (Russian) [8], 68\% (Lithuanian) [9]. When looking at these results, one should keep in mind the aspect that Twitter entries contain a lot of noise, including spam, the short communication style adopted by users, and irrelevant content. This makes sentiment analysis from Twitter and other social media platforms a challenging task [10]. More than half of tweets do not contain opinion or are neutral [10], [11] and also human inter-annotator agreement is low [12] or fair to moderate [6], [11]. These results show that achieving $100 \%$ accuracy is not possible even for humans and $80 \%$ accuracy for automated classifiers should be considered as very high.

During the review of related work three resources were identified as considering opinion mining in Latvian text. The first one is the study by The Institute of Mathematics and Computer Science, University of Latvia [13] with the aim of identifying aggressive comments in contemporary online Latvian language. During this study, data used for the experiments was gathered via project Virtual Aggression Barometer [14] and a normalization tool and an automated classifier were used to detect internet user comments with aggressive and nonaggressive sentiment. During the study the overall accuracy of $72.2 \%$ was achieved: $28.9 \%$ for aggressive comment identification and $85.7 \%$ for non-aggressive comment identification. A list of Latvian stop-words (words that can be ignored during feature selection of the document as they represent little or no opinion information, e.g., prefixes, connectives, and prepositions) was published as a part of this research. The list is also used during our study (further referred as LUMI stop-words).

In the second study [11] researchers used several publicly available machine translation and sentiment analysis systems to detect sentiment tweets in Latvian. During this study the corpora of 1722 manually labeled tweets (labeled positive, neutral or negative) were produced for tests [15]. Their labeled tweet corpus further referred as PS dataset, and results are used as a benchmark for testing results of this study. 
The third identified resource related to opinion mining in Latvian is the list containing 3595 negative sentiment words and 1873 positive sentiment words [16] (further referred as AP lexicon).

More extensive review on related work in opinion mining in non-English texts has been written by the author in [17]. In this work the metrics among the techniques and the results of other studies have also been compared.

\section{Research Method and Tasks}

During the study of related work authors did not identify other studies that would use an extensive lexicon of Latvian text polarity detection or machine learning. Relevant similar work has been done for detecting hateful comments in the internet web pages [13], [14]. In our study we broaden the aim also to detect text with positive polarity. To perform automatic opinion mining of Latvian text, the general approach illustrated by authors of [18] is used. It consists of 6 consecutive steps: Data selection, Method selection, Pre-processing, Transformation, Analysis and Evaluation. The steps, identified challenges and tasks performed during our research are detailed in Table 1.

Table 1. Research steps, tasks, challenges and approaches

\begin{tabular}{|c|c|c|c|}
\hline Research step & Generic tasks & $\begin{array}{l}\text { Challenges related to the } \\
\text { Latvian language }\end{array}$ & Specific tasks \\
\hline \multicolumn{4}{|l|}{ 1. Data selection } \\
\hline & $\begin{array}{l}\text { Select data for } \\
\text { labeling and analysis }\end{array}$ & $\begin{array}{l}\text { Availability of labeled datasets in } \\
\text { Latvian }\end{array}$ & $\begin{array}{l}\text { Use PS dataset } \\
\text { Use Twitter API and build new tweet dataset } \\
\text { in Latvian \& label manually }\end{array}$ \\
\hline & Select 'support' data & $\begin{array}{l}\text { Availability of stop-word list in } \\
\text { Latvian } \\
\text { Availability of sentiment lexicon } \\
\text { in Latvian }\end{array}$ & $\begin{array}{l}\text { Use LUMI stop-words, transform stop-words } \\
\text { into appropriate data structure } \\
\text { Use AP lexicon, translate from EN, review } \\
\text { and add more words from MPQA [22], inflect } \\
\text { words, add emoticons and emoji, transform } \\
\text { lexicon into appropriate data structure }\end{array}$ \\
\hline \multicolumn{4}{|l|}{ 2. Method selection } \\
\hline & $\begin{array}{l}\text { Select classification } \\
\text { approaches }\end{array}$ & $\begin{array}{l}\text { Effectiveness and transparency } \\
\text { of selected methods }\end{array}$ & $\begin{array}{l}\text { Apply methods seen in related work, } \\
\text { especially used in similar work in non-English } \\
\text { Languages } \\
\text { Use simple lexicon-based method } \\
\text { Use 'noisy-labeling' and Naïve Bayes }\end{array}$ \\
\hline & Select tools & Select appropriate tools & Use Excel, Python-nltk \\
\hline \multicolumn{4}{|l|}{ 3. Pre-processing } \\
\hline & Duplicate removal & Identify and remove duplicates & Use custom scripts \\
\hline & $\begin{array}{l}\text { Removal of common } \\
\text { words, usernames, } \\
\text { tags }\end{array}$ & $\begin{array}{l}\text { Identify and remove common } \\
\text { words }\end{array}$ & Use custom scripts \\
\hline & Tokenization & Correctly split text into n-grams & Use 'bag-of-words' approach \\
\hline \multicolumn{4}{|l|}{ 4. Transformation } \\
\hline & $\begin{array}{l}\text { Create 'bag-of-words' } \\
\text { structures }\end{array}$ & $\begin{array}{l}\text { Create appropriate labeled } \\
\text { datasets for ML classifier } \\
\text { training and testing }\end{array}$ & $\begin{array}{l}\text { Split text into unigrams using custom scripts } \\
\text { Create noisy-labeled dataset }\end{array}$ \\
\hline \multicolumn{4}{|l|}{ 5. Analysis } \\
\hline & $\begin{array}{l}\text { Calculate and display } \\
\text { core metrics }\end{array}$ & $\begin{array}{l}\text { Obtain trustable and illustrative } \\
\text { metrics }\end{array}$ & $\begin{array}{l}\text { Using Python-nltk, custom scripts, Excel run } \\
\text { a) Linguistic orientation test } \\
\text { b) Naive Bayes classifier test }\end{array}$ \\
\hline \multicolumn{4}{|l|}{ 6. Evaluation } \\
\hline & $\begin{array}{l}\text { Compare results from } \\
\text { multiple approaches }\end{array}$ & $\begin{array}{l}\text { Select representative metrics } \\
\text { and compare results }\end{array}$ & $\begin{array}{l}\text { Compare with results obtained by other } \\
\text { researchers as reviewed in related work }\end{array}$ \\
\hline
\end{tabular}


Data selection. One of the main reasons why opinion mining tasks for texts in English are easier than in less widely spoken languages, such as Latvian, is the availability of labeled text corpora. In English language resources, with thousands of labeled words, phrases and n-grams are available [20], [21], [22]. We used a labeled tweet dataset by Peisenieks and Skadins (PS dataset) [11] and Twitter API [23] to obtain an additional dataset of tweets and labeled it. The LUMI stop-word list was used for stop-words as support data. AP lexicon was used and extended during the research.

Method selection. Based on the review of related work we selected the following methods during the study:

- Sentiment lexicon, counting positive and negative sentiment words.

- Supervised machine learning classifier training using Naive Bayes (NB) algorithm.

NB was chosen as the particular classification algorithm. Despite the simple assumptions that underlie the NB classifier, experiments on real-world data in this domain have shown it to be competitive with much more sophisticated induction algorithms [7], [9], [24]. To obtain larger dataset for classifier training two approaches were attempted, namely, (1) use of tweets autolabeled by using a lexicon, and (2) noisy labeling of a tweet corpus. The noisy labeling approach is commonly used when researchers lack a readily available text corpus or want to create one that is newly labeled. Its essence is that some very obvious labels, e.g., smiley emoticons are used as the labels to rely on when deciding if particular text belongs to a particular class, e.g., is positive or negative. Noisy labeling approach was also used during this study, and a text corpus with 5556 tweets was created.

Pre-processing and Transformation. Custom scripts were used to remove duplicates and other information, such as mentions, hashtags, URLs from text that is used for the training classifier. To avoid complexity related to language semantics and morphology, a simple 'bag-ofwords' approach was applied as it is also commonly done in other studies [7], [8], [11]. A bagof-words approach perceives text and parts of it as a simple set of words and does not consider relations among such words.

Analysis. During the analysis stage, custom scripts and Excel were used to automatically classify tweets as positive, neutral or negative and aggregate core metrics (accuracy, precision, recall and F-score). Custom Python script and MS Excel were used to count and detect each class of tweet during the first test. Naive Bayes algorithm from Python Natural Language Toolkit (Python-nltk) was used for the second test. Natural Language Toolkit (NTLK) [25] is an open source library, multiplatform collection of tools and modules for natural language processing and text analysis and is distributed under Apache 2.0 license. It provides a large set of tools and algorithms for text tokenization, stemming, tagging, chunking, parsing, classification, clustering, measurement and semantic interpretation.

Evaluation. Testing efficiency of the word polarity lexicon is performed using labeled PS dataset. The results obtained during experiments are compared with each other and with results of similar studies reviewed in the Section 2.

\section{Performing Semantic Polarity Analysis on Latvian Text}

Extending word polarity lexicon in Latvian. During the review of related work it was discovered that some valuable public resources exist that can be used for building an improved word polarity lexicon. During our research we processed these resources and gathered a more extensive lexicon in Latvian. The result of combining these resources for the creation of this extended lexicon is illustrated in Table 2 and published in [26]. 
Table 2. Word polarity lexicon sources

\begin{tabular}{|l|l|l|}
\hline \multicolumn{1}{|c|}{ Resource } & \multicolumn{1}{|c|}{ Words with positive polarity } & Words with negative polarity \\
\hline AP lexicon & 1883 & 3594 \\
\hline $\begin{array}{l}\text { Words from translated MPQA sentiment } \\
\text { word list [20] }\end{array}$ & 215 & 890 \\
\hline $\begin{array}{l}\text { New word forms created using } \\
\text { www.letonika.lv word inflector }\end{array}$ & 30172 & 50037 \\
\hline Emoticons and Emoji & 19 & 13 \\
\hline SIZE OF NEW LEXICON & 30904 & 51490 \\
\hline
\end{tabular}

In the next step testing was performed by using script that takes tweets from PS dataset, line by line, and counts the amount of positive and negative words (according to built lexicon) in each tweet. This script outputs a positive or negative score for each tweet that is summarized (positive score minus negative score). Tweets with a score greater than 0 are evaluated as positive. Tweets with a score less than 0 are evaluated as negative. Tweets with a score 0 are evaluated neutral. Also the value assigned by human annotators is displayed in the spreadsheet.

Semantic polarity analysis experimental results. The results of experiments with semantic polarity analysis on texts in Latvian are summarized in Table 3.

Table 3. Latvian extended lexicon compared with machine-translated text with sentiment detection tools for English language

\begin{tabular}{|l|l|l|}
\hline \multicolumn{1}{|c|}{ Metric } & Extended lexicon & Google Translate + Textalytics \\
\hline Neutral & & \\
\hline Precision & 0.69 & 0.2 \\
\hline Recall & 0.59 & 0 \\
\hline F1 & 0.64 & 0.01 \\
\hline Positive Sentiment & & \\
\hline Precision & 0.54 & 0.64 \\
\hline Recall & 0.68 & 0.7 \\
\hline F1 & 0.60 & 0.67 \\
\hline Negative Sentiment & & \\
\hline Precision & 0.52 & 0.62 \\
\hline Recall & 0.47 & 0.39 \\
\hline F1 & 0.49 & 0.48 \\
\hline Average accuracy & 0.73 & 0.61 \\
\hline
\end{tabular}

From the experimental results we can conclude that for the neutral class the lexicon based method significantly outperforms machine-translated text with sentiment detection tools for English language. The apparent reason for that is the tuning of particular tools with strong bias towards other (Positive/Negative) classes. For other classes the practical application of the lexicon based method closely matches the performance of the one that can be obtained with machine translation solutions and commercial tools for the sentiment polarity detection in English. Also, in practical settings, having an adjustable lexicon gives the user more control and ability to tweak the opinion mining process quickly - e.g., easily and quickly understand why some text was classified in a particular way; as opposed to comparatively lower control over an actual machine translation process and external sentiment polarity detection tool. 
The overall accuracy obtained during our study using an extended lexicon (73\%) was slightly higher than reached by the authors of [14]. Other researchers performing Latvian text classification by using machine translation and known sentiment detection tools for English [11] have also obtained similar accuracy; $54.7 \%-76.0 \%$. However, the tools and approach they apply are more complicated.

\section{Performing Machine Learning Approach on Latvian Text}

Building a noisy-labeled tweet corpus. To build a noisy-labeled tweet corpus in Latvian, the following approach was applied. To collect data Twitter API [21] was used to obtain more than 90000 tweets in Latvian during two weeks. Afterwards tweets were filtered, pre-processed and added to the marked tweet corpus using the following convention:

- Labels used for positive sentiment (POS) - :) / :)) / :))) / :d / ;) / <thumbsup-emoji $>$ / $<$ laugh-emoji $>$ / <smile-emoji $>$ /

- Labels used for negative sentiment (NEG) - : ( / :( / < sad-emoji $>$ / <thumbsdown-emoji $>$ /

- For neutral sentiment (NEU) - phrases scored 0 using a positive/negative word lexicon

To balance the set regarding the negative sentiment class, additional negative tweets were marked and added manually, resulting in a corpus of noisy-labeled tweets with 5556 entries. Classes of the resulting tweet corpus are illustrated in Figure 1.

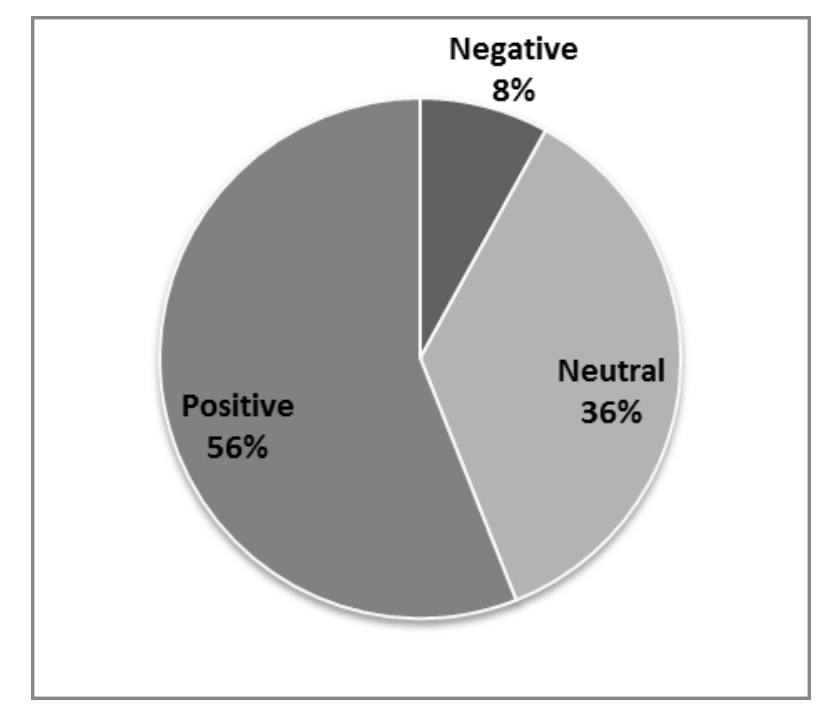

Figure 1. Distribution of classes in noisy-labeled text corpus

Naive Bayes classification experimental results. As stated in Section 3, the well-known Naive Bayes algorithm, which is one of the simplest and also the most commonly used generative classifiers, was applied in order to build a classifier. The authors used Python-nltk implementation of Naive Bayes algorithm.

Considering the relatively small size of the available dataset a test with 10 -fold validation was performed (PS dataset was divided into 10 groups and tested 10 times, each time using 9 different groups for training and one remaining group for testing). Macro-averages of accuracy, precision, recall and F-measure were calculated (see Table 4).

During the tests of the noisy labeled dataset the features that were used for labels (emoticons and emoji) were removed to avoid overfitting. Test results show that the accuracy by using NB classifier is moderate $(62 \%, 55 \%)$, especially measurements regarding negative sentiment. However, a notable advantage is the classifier's ability to automatically track down the most 
informative features (as these words and symbols are used to express positive opinion). They are summarized in Table 5.

Table 4. NB classifier performance with PS and noisy-labeled dataset

\begin{tabular}{|l|c|c|}
\hline \multirow{2}{*}{ Metric } & PS dataset & Noisy-labeled dataset \\
\cline { 2 - 3 } & 10-Fold validation & $\begin{array}{c}\text { Train: Noisy-labeled dataset, } \\
\text { Test: PS dataset }\end{array}$ \\
\hline Neutral & & 0.61 \\
\hline Precision & 0.75 & 0.66 \\
\hline Recall & 0.75 & 0.63 \\
\hline F1 & 0.74 & 0.47 \\
\hline Positive Sentiment & & 0.61 \\
\hline Precision & 0.62 & 0.53 \\
\hline Recall & 0.57 & \\
\hline F1 & 0.54 & 0.80 \\
\hline Negative Sentiment & & 0.02 \\
\hline Precision & 0.21 & 0.04 \\
\hline Recall & 0.20 & 0.55 \\
\hline F1 & 0.22 & \\
\hline Average accuracy & 0.62 & \\
\hline
\end{tabular}

Table 5. Most informative features of PS and noisy-labeled dataset

\begin{tabular}{|c|c|c|}
\hline & PS dataset & Noisy-labeled dataset \\
\hline $\begin{array}{l}10 \text { most informative } \\
\text { features }\end{array}$ & $\begin{array}{l}:(\text { - negative } \\
:) \text { - positive } \\
\text { vairs - negative (en - anymore) } \\
:)) \text { - positive } \\
\text { nevaru - negative (en - can't) } \\
\text { brīvdienas - positive (en - } \\
\text { holydays) } \\
\text { jütos - negative (en - I am feeling) } \\
\text { nevar - negative (en - can't) } \\
\text { auksti - negative (en - cold) } \\
\text { paldies - positive (en - thank you) }\end{array}$ & $\begin{array}{l}\text { nevaru - negative (en - I can't) } \\
\text { stulbi - negative (en - stupid) } \\
\text { labi - positive (en - good) } \\
\text { nevar - negative (en - can't) } \\
\text { prieks - positive (en - happiness) } \\
\text { slikts - negative (en - bad) } \\
\text { forši - positive (en - cool) } \\
\text { labs - positive (en - good (male gender)) } \\
\text { laba - positive (en - good (female gender)) } \\
\text { izrādās - negative (en - it appears) }\end{array}$ \\
\hline
\end{tabular}

By further automating the classification process, adding iterative steps of feedback to the classifier, and training it on a larger dataset, it is possible to create more accurate classifiers. There are attempts to design interactive classification systems, which can benefit applications where the initially available training set is insufficient and a human expert is available [27]. This could also be one of the directions for obtaining higher accuracy in opinion mining.

\section{Conclusions and Future Work}

Approaches used in other languages are viable for opinion mining in Latvian. Even the application of basic opinion mining methods can give reasonable results (e.g., F1 score during our tests with Extended lexicon was 12 percentage points higher for negative sentiment, 7 percentage points lower for positive sentiment and 63 percentage points higher for Neutral sentiment than F1 score for Google Translated text with Textalytics sentiment detection tool obrained by [11]). However, precision and recall, especially for negative class detection observed during the tests is rather low. Our experiments show that lexicon-based methods provide more accurate opinion mining than the application of Naive Bayes machine learning classifier on Latvian tweets. However, machine learning retains the ability to detect most informative features of the tweets thus producing new human-readable knowledge about the 
domain. Further development of the resources for opinion mining in Latvian is necessary to build better opinion mining classifiers. At present the lack of large annotated text sets hinders attempts to build machine learning classifiers with reasonably high accuracy. Methods used in this study could be further improved to obtain results comparable with current results in opinion mining in English and other languages. The development and publishing of resources [26] as annotated datasets, a Latvian lexicon (containing 30904 positively labeled words and 51490 negatively labeled words), algorithms and methods for opinion mining, may further foster development of commercially useable applications for practical use in specific domains or particular organizations and/or studies for organizations in Latvia or international organizations interested in aggregating opinions of the Latvian speaking population.

Some particular directions for future work and improvements are the following:

- Significantly increase the existing set of noisy-labeled tweets. Possibly wider use of emoticons as 'noisy labels' and retrieving more significant amounts of relevant data from social networks.

- Implement better emoji and emoticon support (particularly - tokenization) in tools, training data and dictionaries.

- Add the scoring of positivity and negativity to the lexicon as some words or phrases are more positive/negative than others, e.g., using an approach similar to MPQA lexicon [22] of positive and negative English words.

- Develop more interactive ways for lexicon management.

- Development of commercial applications (e.g., subscription based service for monitoring Latvian content in Social Networks related to a particular subject).

\section{References}

[1] W. Medhat, A. Hassan and H. Korashy, "Sentiment Analysis Algorithms and Applications: A Survey," in Shams Engineering Journal, vol. 5, no. 4, pp. 1093-1113, $2014 . \quad$ Available: http://dx.doi.org/10.1016/j.asej.2014.04.011

[2] B. Pang and L. Lee, "Opinion Mining and Sentiment Analysis," Foundations and trends in information retrieval, vol. 2., no. 1-2, pp. 1-135, 2008. Available: http://dx.doi.org/10.1561/1500000011

[3] Google, Google Scholar, 2016. [Online]. Available: https://scholar.google.com/scholar?hl=en\&q=opinion+ mining (accessed March 23, 2016)

[4] S. Volkova, T. Wilson and D. Yarowsky, "Exploring Sentiment in Social Media: Bootstrapping Subjectivity Clues from Multilingual Twitter Streams," in ACL, vol. 2, pp. 505-510, August, 2013.

[5] Y. Chen and S. Skiena, "Building Sentiment Lexicons for All Major Languages," in Proc. the 52nd Annual Meeting of the Association for Computational Linguistics, pp. 383-389, 2014. Available: http://dx.doi.org/10.3115/v1/p14-2063

[6] S. Narr, M. Hulfenhaus and S. Albayrak, "Language-independent Twitter Sentiment Analysis" in Knowledge Discovery and Machine Learning (KDML), LWA 2012, pp. 12-14, 2012. [Online]. Available: http:// www.dai-labor.de/fileadmin/Files/Publikationen/Buchdatei/narr-twittersentiment-KDML-LWA-2012.pdf

[7] P. A. Tapia and J. D. Velásquez, "Twitter Sentiment Polarity Analysis: a Novel Approach for Improving the Automated Labeling in a Text Corpora," in Active Media Technology, Springer International Publishing, pp. 274-285, 2014. Available: http://dx.doi.org/10.1007/978-3-319-09912-5_23

[8] I. Chetviorkin, L. G. Moscow and N. Loukachevitch, "Two-Step Model for Sentiment Lexicon Extraction from Twitter Streams," ACL 2014, 67, 2014. [Online]. Available: http://anthology.aclweb.org/W/W14/W14-26.pdf\#page=79

[9] A. Krupavicius and T. Krilavicius, "A Comparison of Approaches for Sentiment Classification on Lithuanian Internet Comments” ACL 2013, vol. 2, 2013.

[10] A. Hassan, A. Abbasi and D. Zeng, "Twitter Sentiment Analysis: A Bootstrap Ensemble Framework," in Social Computing (SocialCom), 2013 International Conference on, IEEE, pp. 357-364, 2013. Available: http://dx.doi.org/10.1109/socialcom.2013.56 
[11] J. Peisenieks and R. Skadiňš, "Uses of Machine Translation in the Sentiment Analysis of Tweets," Human Language Technologies - The Baltic Perspective, pp. 126-131, 2014. Available: http://dx.doi.org/10.3233/978-1-61499-442-8-126

[12] J. R. Landis and G. G. Koch "The Measurement of Observer Agreement for Categorical Data," Biometrics, pp. 159-174, 1977. Available: http://dx.doi.org/10.2307/2529310

[13] G. Garkāje, E. Zilgalve and R. Darğis "Normalization and Automatized Sentiment Analysis of Contemporary Online Latvian Language.” in Baltic HLT, pp. 83-86, 2014. Available: http://dx.doi.org/10.3233/978-1-61499-442-8-83

[14] Virtual Aggression Baromete, 2014. [Online]. Available: http://barometrs.korpuss.lv/ (accessed March 20, 2015)

[15] Latvian tweet sentiment corpus, 2014. [Online]. https://github.com/FnTm/latvian-tweet-sentiment-corpus (accessed March 20, 2015)

[16] A. Pumpurs, Lexicon of Positive and Negative Sentiment Words in Latvianin in GitHub, 2014. [Online]. Available: https://github.com/pumpurs/SentimentWordsLV (accessed March 12, 2015)

[17] G. Špats, Application of Opinion Mining for Written Content Classification in Latvian Text, Master Thesis, Riga Technical University, 2015.

[18] G. Petz, M. Karpowicz, H. Fürschuß, A. Auinger, S. M. Winkler, S. Schaller and A. Holzinger, "On text preprocessing for opinion mining outside of laboratory environments," in Active media technology, Springer Berlin Heidelberg, pp. 618-629, 2012. Available: http://dx.doi.org/10.1007/978-3-642-35236-2_62

[19] P. Faschang, G. Petz, V. Dorfer, T. Kern and S. M. Winkler, “An Approach to Mining Consumer's Opinion on the Web," in 13th International Conference on Computer Aided Systems Theory, Eurocast 2011, pp. 37 39, 2011.

[20] H. Saif, M. Fernandez, Y. He and H. Alani, "Evaluation Datasets for Twitter Sentiment Analysis: a Survey and a New Dataset, the STS-Gold." 2013. [Online]. Available: http://oro.open.ac.uk/40660/1/paper1.p

[21] A. Esuli and F. Sebastiani, "Sentiwordnet: A publicly Available Lexical Resource for Opinion Mining," in Proc. the LREC, vol. 6, pp. 417-422, 2006.

[22] University of Pittsburgh, MPQA Opinion Corpus, 2015. [Online]. Available: http://mpqa.cs.pitt.edu/ (accessed May 3, 2015)

[23] Twitter, Twitter API documentation, 2015. [Online]. Available https://dev.twitter.com/overview/api (accessed December 20, 2015)

[24] G. H. John and P. Langley, "Estimating Continuous Distributions in Bayesian Classifiers," in Proc. the Eleventh conference on Uncertainty in artificial intelligence, Morgan Kaufmann Publishers Inc., pp. 338$345,1995$.

[25] NLTK Project, NLTK 3.0 documentation, 2016. [Online]. Available: http://www.nltk.org/ (accessed March 5, 2016)

[26] G. Špats, Resources for Opinion Mining in Latvian, 2015. [Online] Available: https://github.com/gatis/om (accessed March 25, 2016)

[27] I. Birzniece, P. Rudzajs and D. Kalibatiene, "Evaluating the Application of Interactive Classification System in University Study Course Comparison," in 11th International Conference on Perspectives in Business Informatics Research, Springer LNBIP, pp. 335-346, 2014. Available: http://dx.doi.org/10.1007/978-3-319-11370-8_24 